TP Periodica Polytechnica Architecture

47(2), pp. 119-125, 2016

DOI: 10.3311/PPar.10385

Creative Commons Attribution (i)

RESEARCH ARTICLE

\section{Information and Communications Technology in Construction: A Proposal for Identifying the Root Causes of CPI}

\author{
Pablo Orihuela $^{1,2^{*}}$, Jorge Orihuela ${ }^{2}$, Santiago Pacheco ${ }^{1,2}$
}

Received 09 December 2016

\begin{abstract}
To improve feedback from the Cost Performance Index (CPI), it is necessary to identify the reasons why Actual Cost differs from Planned Cost. At present, this information is collected and transferred orally, on paper, by telephone or e-mail. This paper proposes an information and communication technology that uploads information to an Intranet using electronic devices and mobile applications, thus encouraging the participation of workers and other stakeholders. This proposal aims to contribute towards information quality improvement in Production Control.
\end{abstract}

\section{Keywords}

automation, Information Systems in Construction, ICT in construction, Production Control, Earned Value Management

\section{Introduction}

If we want to talk about improvement of either companies or businesses from any field, we necessarily need to revisit Kaizen or Continuous Improvement; this proposes putting into practice the Shewhart Cycle, better known as the Deming Cycle in Japan since it was Dr Deming who made it public, or the PDCA Cycle (Plan-Do-Check-Act) (Walton, 1988; Imai M., 1986; Liker, 2013).

In the civil construction field, applying this cycle consists of planning, execution, evaluation, and corrective measures for improvement; however, in practice, the third stage - the one pertaining to evaluation - is not successfully complied with. This is because, significantly, information about the use of work resources is not reliable or is not available when necessary (Dave et al., 2010).

This article proposes an information and communication system to collect data directly from the worksite and process it on the Web, consequently, it will be possible to evaluate and control all construction site activities on a continuous basis, having access to it from anywhere. This system was already patented by its authors several years ago, with excellent results, but what we are incorporating in this new proposal is the technology to automatize both data collection and processing. Said system collects data from the three resources of production through electronic devices and process it with online software available in a Web application.

The labour resource is reported by the workers themselves using touch screens in the late morning, and in the late afternoon at the end of each shift. Material consumption is recorded online by tracking the materials leaving the warehouse and being subsequently used. The use of equipment is registered by the operators. Finally, work progress is reported online on a daily basis from their worksite through digital tablets using store-and-forward applications.

\footnotetext{
${ }^{1}$ Pontificia Universidad Católica del Perú, Lima 15088, Peru

${ }^{2}$ Motiva S.A., Lima 15073, Peru

*Corresponding author, e-mail: porihuela@motiva.com.pe
} 


\section{Managing Production Information in Construction Works}

The quality of production information in construction works is not consistent with current times; in practice, we can only obtain accurate information on the costs at the end of the works. Apart from being late, the information delivered by traditional control systems is too grouped to be useful for controlling and planning decision making (Marchesan and Formoso, 2001; Navon and Sacks, 2007).

In the last two decades, the construction industry has shown great advances in the use of ICT worldwide, even in small and medium-sized enterprises, as described by several authors (Howard et al., 1998; Tas and Irlayici, 2007; Michaloski and Cabral, 2010; Alaghbandrad et al., 2011; Abuelmaatti et al., 2014; Ibem and Laryea, 2015). However, as Dave et al. (2008) conclude, based on the work of Tartari et al. (2007), the "majority of ICT solutions within the construction industry are applied to the peripheral processes" and "site management and other construction related activities have remained virtually unaffected." Additionally, 90\% of ICT expenses are incurred in the technical work office or head office and only $10 \%$, for field use (Anon., 2016).

This is consistent with Bowden's studies (Bowden, 2002; Bowden et al., 2004), which presents the existence of 85 paper-based tasks carried out on-site as part of their normal daily work. "These were grouped into different document types revealing the most commonly identified tasks as completing data collection forms $(25 \%)$, dealing with correspondence $(18 \%)$, viewing and reviewing drawings (13\%) and reading and writing specifications $(6 \%)$ ".

From the research conducted in the UK by Chen and Kamara, it can be concluded that the information necessary for production monitoring (materials, labour, equipment and progress) is collected using paper-based forms (65\%); by remembering the information (28\%); and through mobile devices (only $7 \%$ ), as can be seen in Fig. 1. Also, as shown in Fig. 2, transfer of the information collected is done mainly in face-to-face meetings ( $42 \%)$, by e-mail (31\%) or phone ( $20 \%)$, and only $7 \%$ is sent over the Intranet or Extranet (Serpell, 2002).

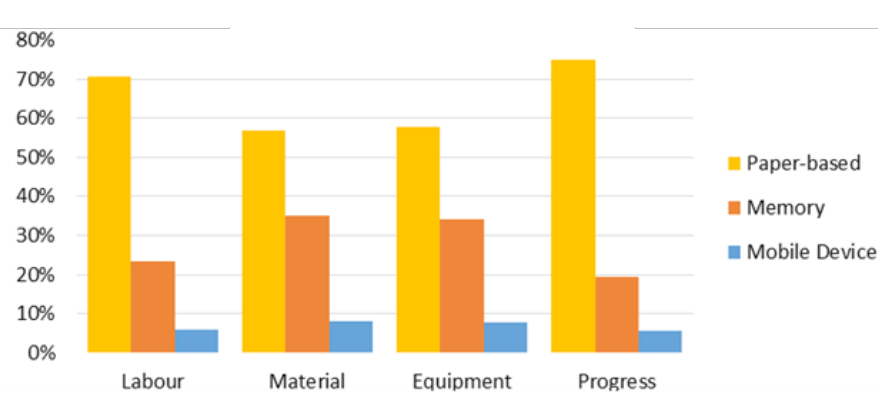

Fig. 1 Production information collection methods.

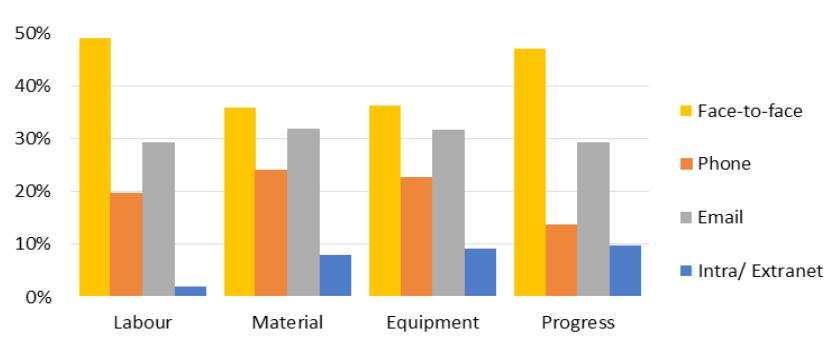

Fig. 2 Production information transfer mediums

The results presented demonstrate the inefficient manner in which production information is handled on the construction site and the existence of great improvement opportunities. The Information Technology and System proposed in this paper eliminates the need to fill out information collection forms about the use of production resources during the information collection process. In addition, this information is entered directly into a Web application, so it does not require an additional information transfer process.

\section{Continuous Improvement in Production Control}

Production Control must be planned within the framework of Continuous Improvement. If control activities indicate that we are doing something wrong, it is necessary to identify the problem to solve it. Likewise, if we are getting good results, it is necessary to determine which the effective practices are to standardise them. Therefore, the control in the Check stage of the PDCA Cycle must provide enough information to identify why those results were obtained and act accordingly, thus closing the cycle.

To implement Continuous Improvement in Production Control, the Lean Construction Institute proposes using the Last Planner System ${ }^{\circledR}$. For the first stage (Plan), they suggest following this sequence: Master Schedule, Phase Schedule, Look Ahead Plan, and Weekly Work Plan (Fig. 3a). After the Weekly Work Plan has been executed (Do), the Percentage of Plan Complete (PPC) and the Reasons for Non-Compliance (RNC) must be verified (Check) to complete the learning process. However, these indicators only shed light on the efficiency of the program, not on the costs.

On the other side, for Continuous Improvement, the Project Management Institute recommends the Earned Value Technique (Fig. 3b) in its PMBOK Guide. First, Planned Value (Plan) must be estimated, and then Earned Value and Actual Costs (Do) must be calculated at the end of any control period. After obtaining these three values, we calculate (Check) the Cost Performance Index (CPI) and the Schedule Performance Index (SPI) and then act accordingly. Nonetheless, these indicators are very broad, and we consider that CPI should also include some RNC like PPC does. 
a)

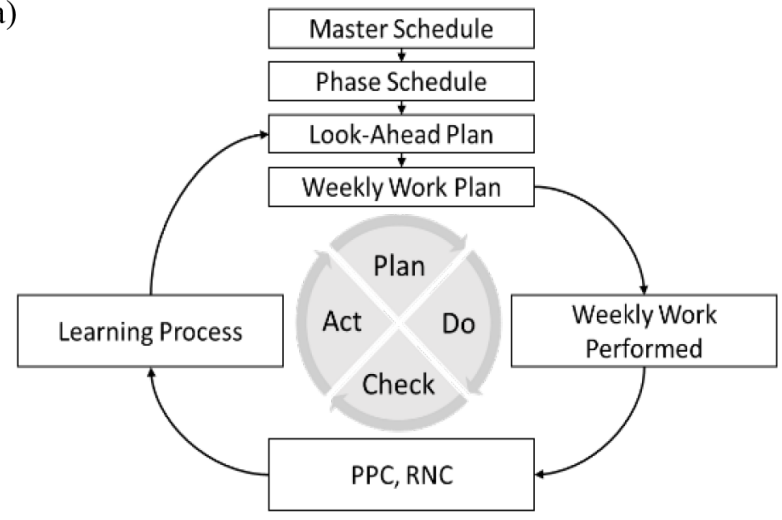

b)

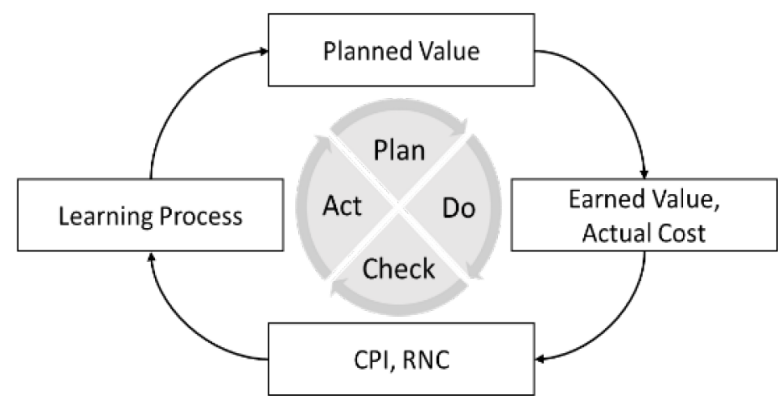

Fig. 3 (a) The Last Planner System ${ }^{\circledR}$ and Continuous Improvement; (b) Earned Value and Continuous Improvement.

Thus, it is necessary to make a Root Cause Analysis of the Actual Cost and apply the 5 Whys Technique, with which we will obtain a Tree Diagram as shown in Fig. 4. Here, the seven root causes that can make the Actual Cost differ from the Earned Value are displayed.

\section{The Seven Groups of Causes for Non-Compliance with the Actual Cost}

Productivity in construction works poses a complex problem due to the number of variables involved. Therefore, it is important to know which factors are negatively or positively affecting the executed tasks to address them (McGregor, 1960). In this scenario, it is crucially important to identify the causes and source of variations concerning the planned cost to complete the continuous improvement cycle and start the feedback stage.

Figure 4 also outlines how the Actual Cost of a project is calculated from the savings and losses results of all the tasks, and how this can differ from the Earned Value due to the seven groups of root causes, based on the assumption that the baseline is correct:

Cause No. 1: Difference in the amount of work foreseen for each task due to errors in the calculations done based on the plans during the planning stage. In this case, feedback is directed to the planners.

Cause No. 2: Difference in the number of man-hours used due to a lower or higher workforce productivity, which in turn may have different causes such as lack of space, timely information, security aspects, availability of equipment, tools and materials, and motivation levels.
Cause No. 3: Difference in the number of machine hours used, which may be due to workforce productivity and machine performance.

Cause No. 4: Difference in the amount of materials used, which may be due to waste surpassing regular standards, overdosing, oversized materials, and reworking.

Causes No. 5, 6, and 7: Price differences concerning workforce, equipment or materials, which chiefly obey market reasons and are mainly controlled by the procurement management.

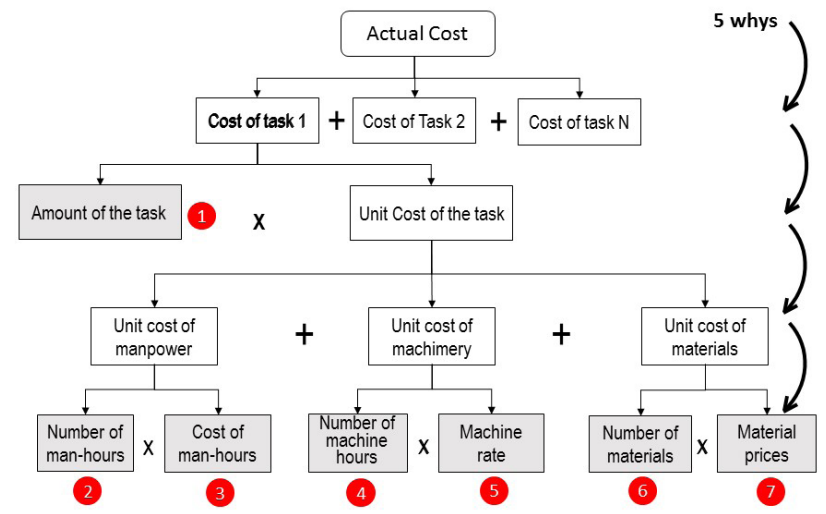

Fig. 4 Root Causes for the difference between Actual Cost and Earned Value.

From the information contained in this section, we can verify that if we want valuable feedback to complete the continuous improvement cycle, the Cost Performance Index (CPI), as shown in Fig. 3, must identify some Causes for Non-Compliance, so we know where to direct all corrective measures.

From these seven causes, three of them are very easy to obtain since they are market prices set for supplies. However, to obtain the remaining four causes, detailed field information is required. This is where our proposed ICT System contributes to Production Control.

\section{Proposed Information Technology and System}

Our proposal focuses on production improvement: The first issue is to improve the quality of poor communication and information between the management, professional and technical staff, and the productive entity by building a bridge that efficiently completes the feedback-based improvement cycle.

The proposed information collection is described below:

\subsection{Defining the Baseline}

Before starting the construction work, it is necessary to define the baseline that production control and tracking will be compared to. This baseline is made up of construction time, cost and scope; therefore, information about work budget, activities schedule and technical specifications must be previously entered in the system. 


\subsection{Collecting Information about the Use of Labour and Equipment}

The main proposal of our system focuses on breaking the deep-seated paradigm, especially in the construction sector, which is managing works according to Theory $\mathrm{X}$. Whereas this theory considers that the workers cannot control themselves, cannot be motivated, and just work for money (Ouchi, 1982), our system supports Theory Z, proposed by William Ouchi, an intermediate point between McGregor's Theory X and Theory $\mathrm{Y}$ [21]. This considers that workers are motived not only by money but also by new challenges and the trust their company may place in them.

By delegating the responsibility for information to the source of production, i.e. the workers themselves, a permanent control is available, not over discretionary samples, but over $100 \%$ of the activities and $100 \%$ of the resources. Therefore, at lunch time and the end of their shifts, workers go to the registration site, identify themselves using a biometric face reader (Fig. 5a), and make a self-report about the time destined to each activity (Fig. 5b) using touch screens.

The Web application developed for this operation was designed to be user-friendly, so it does not pose any obstacle for workers to execute this action. The identification of activities shows images to help them easily identify the activities performed, while the display of images on the screen is automatically filtered according to the worker's profile after his identification. This requires less effort, and it is easier for workers to draw up self-reports about the time spent on each activity.

a)

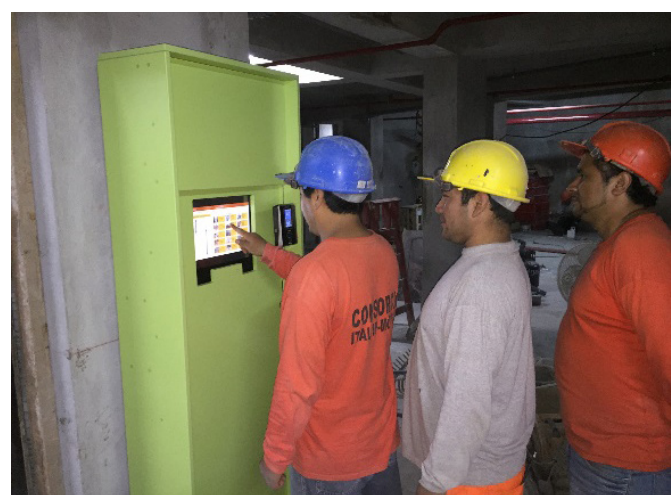

b)

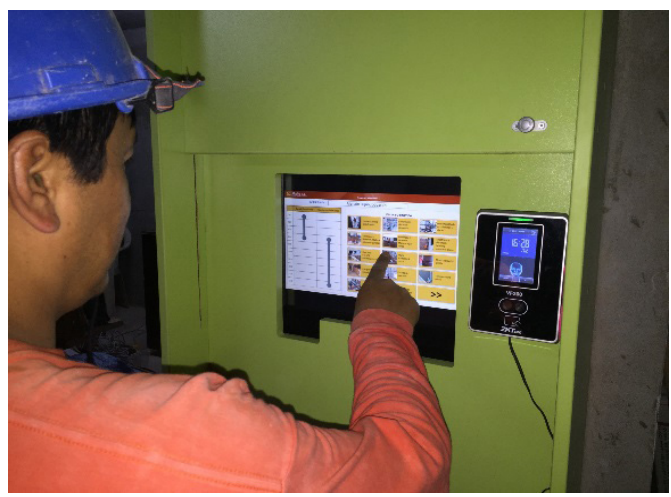

Fig. 5 (a) Worker Identification and self-report;

(b) Worker self-report on a touch screen.
Before entering the worksite, new workers watch a 30-minute training video that easily and readily explains the information system, the importance of this system for the company, and how they are expected to participate. Every new worker must watch this video and take a short guided test on self-reporting. Additionally, workers who have operated equipment or machinery must also make a report, in a similar manner, about how long they have been operating them.

\subsection{Collecting Information about the Use of Materials}

All material that enters the work site must be registered in the warehouse. For this task, the warehouse keeper can access the system to register it on the Web directly, also recording the quantity received and unit cost. Then, when these materials are required to be used, he must also record their exit, showing the quantity and destination record.

\subsection{Collecting Information about the Amount Work Progress}

Due to the nature of production in construction, the amount of work progress is recorded on site, going around the worksite registering information in different places where tasks have been carried out. This information is collected by a person in charge at the end of the workday using a digital tablet (Fig. 6a). This procedure helps save time normally spent transferring the collected data-generally on paper-to a control system, which in most cases is done in an electronic spreadsheet. To avoid the lack of Wi-Fi connection on the worksite, where we record the work progress, becoming an issue, an application for mobile devices with a store-and-forward feature is used. This allows information to be temporarily stored in the device (Fig. 6b) and then automatically uploaded to the Web as soon as a Wi-Fi connection is available.

\subsection{The 7 Groups of CPI Indicators}

Based on the data collected on site; when the cost of a task considerably differs from the baseline, we can ask the system to show the seven groups of indicators for the task at hand, which will consequently guide us to find the root causes for the cost difference. We say, a group of indicators because each one of them will have more than one indicator. They will have as many indicators as there are supplies in each task. For example, for the Groups related to Materials, there will be one indicator for each material; for the Workforce, one for each worker category; and for the Machine, one per each machine used for the task.

Figure 7 outlines the seven groups of indicators for the task we want to analyse. The difference between the actual cost of a task and its planned cost may be due to a combination of effects, which can be demonstrated and analysed in their corresponding indicators. 
a)

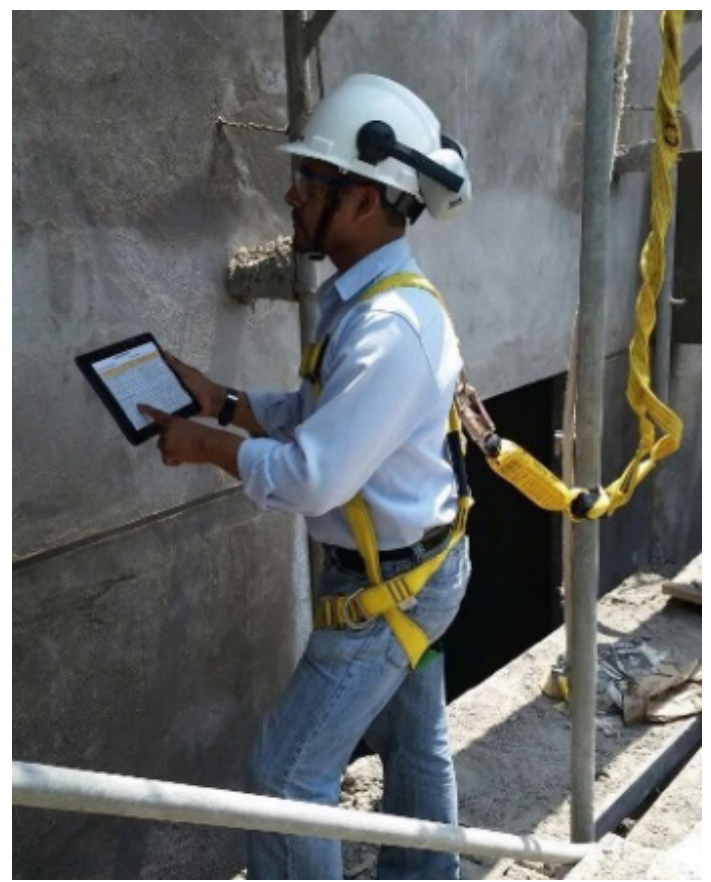

b)

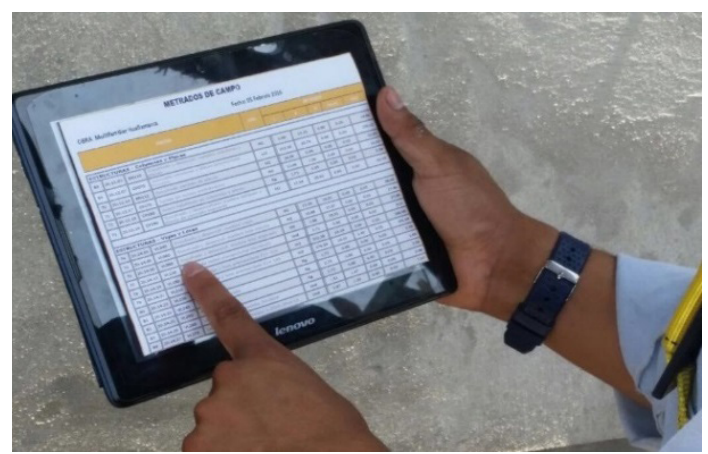

Fig. 6 (a) On-site work progress registration;

(b) Use of digital tablets and store-and-forward.

\begin{tabular}{|c|c|c|c|c|c|c|}
\hline \multirow{3}{*}{$\begin{array}{l}\text { AMOUNT } \\
\text { OF TASK }\end{array}$} & \multicolumn{2}{|c|}{ PLANNED } & \multicolumn{2}{|c|}{ ACTUAL } & \multicolumn{2}{|c|}{ GROUP OF } \\
\hline & \multicolumn{2}{|c|}{$\mathrm{A}_{1}$} & \multicolumn{2}{|c|}{$\mathrm{A}_{2}$} & \multicolumn{2}{|c|}{$\mathbf{I}_{1}=\mathbf{A}_{2} / \mathbf{A}_{1}$} \\
\hline & Amount & Price & Amount & Price & Amount & Price \\
\hline $\begin{array}{c}\text { MAN- } \\
\text { HOURS }\end{array}$ & $\mathrm{A}_{3}$ & $\mathrm{P}_{1}$ & $\mathrm{~A}_{6}$ & $\mathrm{P}_{4}$ & $\mathbf{I}_{2}=\mathbf{A}_{6} / \mathbf{A}_{3}$ & $\mathbf{I}_{3}=\mathbf{P}_{4} / \mathbf{P}_{1}$ \\
\hline $\begin{array}{c}\text { MACHINE } \\
\text { HOURS }\end{array}$ & $\mathrm{A}_{4}$ & $\mathrm{P}_{2}$ & $\mathrm{~A}_{7}$ & $\mathrm{P}_{5}$ & $\mathbf{I}_{\mathbf{4}}=\mathbf{A}_{7} / \mathbf{A}_{4}$ & $\mathbf{I}_{5}=\mathbf{P}_{5} / \mathbf{P}_{2}$ \\
\hline MATERIALS & $\mathrm{A}_{5}$ & $\mathrm{P}_{3}$ & $\mathrm{~A}_{8}$ & $\mathrm{P}_{6}$ & $\mathbf{I}_{6}=\mathbf{A}_{8} / \mathbf{A}_{5}$ & $\mathbf{I}_{7}=\mathbf{P}_{6} / \mathbf{P}_{3}$ \\
\hline
\end{tabular}

Fig. 7 The seven groups of CPI Indicators for an analysis task.

\subsection{Production Reports}

Once the system has all the daily production information uploaded to the Web, several reports will be available to track, control and provide a quite clear idea of the global labour productivity at any given time. The different reports can be accessed at any stage of the works with a one day delay. The performance diagnosis can be obtained within the periods matching the dates when the work progress was recorded, thus reaching a $100 \%$ control of the works without incurring excessive control staff expenses, since the system is practically run by the workers themselves.

By reviewing each user profile, different stakeholders can access varied reports according to their needs. For instance, crew leaders and the foreman can check their staff performance; contractors can track their staff if they also participate in this system; warehouse keepers can check all incoming and outgoing materials as well as valued stock; administrative offices can get updated information on staff assistance to calculate payrolls; professionals involved in the works can check the details concerning schedule, costs and performance of each task; and management can check production ratios, actual results, and forecasts for the end of the works.

Figure 8 shows the traditional information flow corresponding to production in construction works, while Figure 9 shows the same flow with the system we propose if implemented. The benefit obtained can be clearly seen, by simplifying the collection, transfer, storage, processing, distribution and communication of data for the stakeholders.

\section{Contribution of the System for Continuous Improvement}

Feedback in construction works is managed late with approximate figures, isolated samples, and not very trustworthy information, which prevents us from implementing a continuous improvement cycle in an effective manner. Thanks to this system, information is continuous and permanent, and since the control of the activities executed is carried out with the same accuracy as the control of the activities planned, very efficient feedback is received the day after the execution of the works. Additionally, this system shows the root causes behind why the executed activities failed to meet the planned goals and allow corrective measures, learning from problematic issues, modifying of processes, and proposes innovations.

Having this Web-based system makes it possible to share information with all the stakeholders, thus providing feedback in every direction, promoting proposals of continuous improvement supported through collaborative teamwork. An important aspect is that management should be involved. Normally, due to restrictions on time, distance, access, or poor communication, management loses its connection with on-site problems, i.e. it does not get involved or support the continuous improvement programmes for the projects. Conversely, the workers are now contributing to the system in a significant way, making them important agents for project development.

This constant feedback allows us to evaluate innovations since we can compare the new results with the ones obtained before the implementation, and now have a historical data bank for the company, enabling improved planning of future projects. 


\section{Conclusions}

CPI shows how different the Actual Cost is from the Earned Value, but after analysing the root cause of the Actual Cost and applying the 5 Whys Technique, the seven causes behind this difference can be identified. Getting information on three of them is easy because they are market prices; however, for the other four, an information and communication system as the one proposed in this paper is necessary. In this way, the seven performance indicators for each advanced task are identified and the reasons for changes in the Actual Cost compared to the Planned Cost are known in detail.

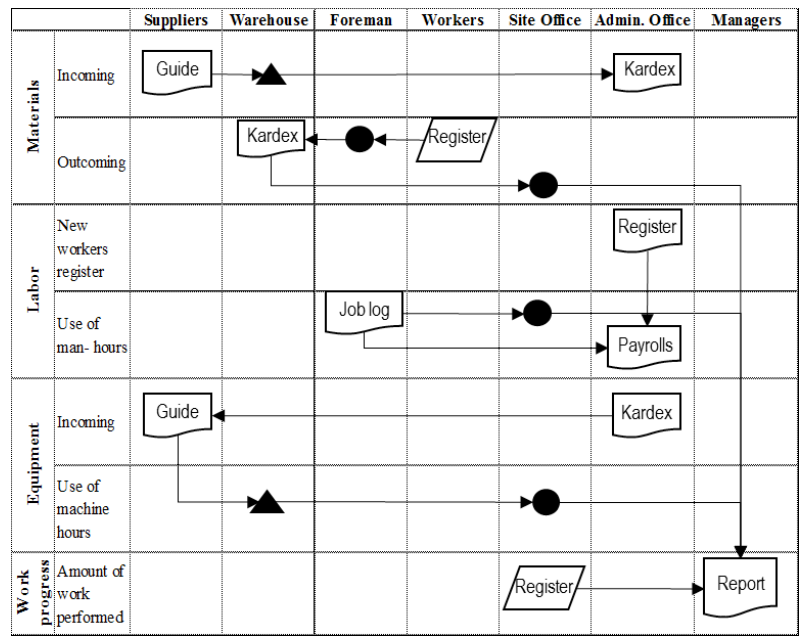

Fig. 8 Traditional production information flow.

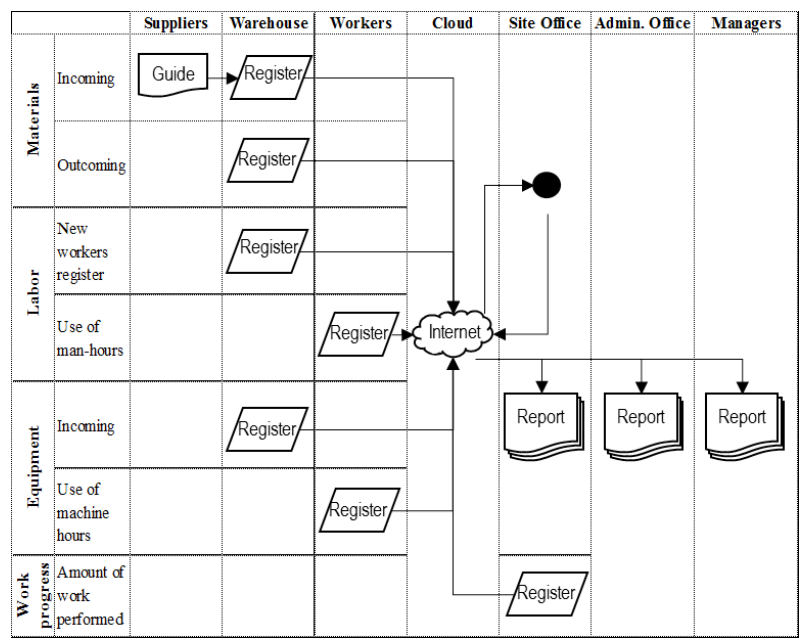

Fig. 9 Production information flow with the proposed system.

The system proposed is based on three main points: 1). Transfer the task to obtain most of the production information to the workers. 2). Use Web applications to promote collaborative work to enter data and distribute shared information. 3). Use electronic devices to make data collection easier.

The first point-considered crucial and questionable — has been a successful daily practice in all the works our company has been carrying out for many years. The second point also shows the same scenario since we already have an intranet containing a module with the presented proposal that is thoroughly approved by all the stakeholders. On the other hand, the third point is an innovation in the pilot stage, which we intend to use to complete the missing link to create an efficient Continuous Improvement Cycle application for the construction sector.

\section{References}

Abuelmaatti, A., Ahmed, V. (2014). Collaborative Technologies for Small and Medium Sized Architecture, Engineering and Construction Enterprises: Implementation Survey. Journal of Information Technology in Construction. 19, pp. 210-224.

Alaghbandrad, A., Asnaashari, E., Preece, C. (2012). Problems and Barriers of ICT Utilization on Iranian Construction Sites: Case Study on the Successful Use of ICT in Remote Construction Sites. Journal of Information Technology in Construction. 17, pp. 93-102.

Anon. (2016). Autodesk - BIM 360 Field, Overview, 2013. [Online]. Available from: http://www.autodesk.com/products/bim-360-field/overview [Accessed: 14 July 2016]

Bowden, S. (2002). The Appropriate Use of IT on a Construction Site. Loughborough University, 2002.

Bowden, S. L., Dorr, A., Thorpe, A., Anumba, C. J. (2004). Mapping site processes for the introduction of mobile IT. In: Proceedings of the 5th European conference on product and process modeling in the building and construction industry, Istanbul, Turkey, Sep 8-10, 2004. (Dikbas, A., Scherer, R. (eds.)), pp. 491-499. https://doi.org/10.1201/9780203023426.pt7

Dave, B., Boddy, S., Koskela, L. (2010). Improving Information Flow within the Production Management System with Web Services. In: 18th Annual Conference of the International Group for Lean Construction, Haifa, Israel, Jul 14-16, 2010. (Walsh, K., Alvarez, T. (eds.)), pp. 445-455.

Dave, B., Koskela, L., Kagioglou, M., Bertelsen, S. (2008). A Critical Look at Integrating People, Process and Information Systems Within the Construction Sector. In: 16th Annual Conference of the International Group for Lean Construction, Manchester, 2008. (Tzortzopoulos, P., Kagioglou, M., (eds.)).

Howard, R., Kiviniemi, A., Samuelson, O. (1998). Surveys of IT in the Construction Industry and Experience of the IT Barometer in Scandinavia. Journal of Information Technology in Construction. 3, pp. $45-56$.

Ibem, E. O., Laryea, S. (2015). E-Procurement Use in the South African Construction Industry. Journal of Information Technology in Construction. 20, pp. 364-384.

Imai M., M. (1986). KAIZEN: The key to Japan's Competitive Success. 259 p. Random House Business Division, New York. 1986.

Liker, J. K. (2013). The Toyota Way: 14 management principles from the world's greatest manufacturer. 2nd Edition, McGraw-Hill, New York. 2013.

Marchesan, P. R. C., Formoso, T. (2001). Cost Management and Production Control for Construction Companies. In: 9th Annual Conference of the International Group for Lean Construction, Singapore, 2001. (Ballard, G., Chua, D. (eds.)).

McGregor, D. (1960). The Human Side of Enterprise. McGraw Hill, New York.

Michaloski, A. O., Costa, A. P. C. S. (2010). A survey of IT use by small and medium-sized construction companies in a city in Brazil. Journal of Information Technology in Construction. 15, pp. 369-390.

Navon, R., Sacks, R. (2007). Assessing research issues in Automated Project Performance Control (APPC). Automation in Construction. 16(4), pp. 474-484. https://doi.org/10.1016/j.autcon.2006.08.001

Ouchi, W. (1982). Theory Z. Avon, New York. 1982. 
Serpell, A. (2002). Administración de Operaciones de Construcción. 296 p. Alfaomega, México D.F., 2002. (in Spanish)

Tas, E., Irlayici, F. P. (2007). A survey of the use of IT in building product information acquisition in Turkey. Journal of Information Technology in Construction. 12, pp. 323-335.

Tatari, O., Castro-Lacouture, D., Skibniewski, M. J. (2007). Current state of construction enterprise information systems: survey research. Construction Innovation. 7(4), pp. 310-319. https://doi.org/10.1108/14714170710780075

Walton, M. (1988). The Deming Management Method. 262 p. Penguin, New York. 